\title{
Combinable Tabs: An Interactive Method of Information Comparison Using a Combinable Tabbed Document Interface
}

\author{
Gonglue Jiang ${ }^{1}$, Chen Zhao ${ }^{2}$, Matthew R. Scott ${ }^{2}$, and Fang Zou ${ }^{2}$ \\ ${ }^{1}$ Zhejiang University, No.38, Zheda Road, Hangzhou, 10027, China \\ jianggonglue@hotmail.com \\ ${ }^{2}$ Microsoft Research Asia \\ 49, Zhichun Road, Haidian District, Beijing 100190, China \\ \{chzha, mrscott, v-fzou\} @microsoft.com
}

\begin{abstract}
The Tabbed Document Interface ("TDI") of today's web browsers is widely considered to be a usability improvement over the previously predominate Single Document Interface ("SDI"). TDI styled interfaces however, especially in mashups or other overlays of different information sets, suffer from a key usability limitation: they cannot show two or more tabbed documents simultaneously. Users are left with the task of quickly switching between tabs when trying to visually compare content. Our contribution is centered on intuitively enabling the comparison of tabbed content via what we coined a Combinable Tabbed Document Interface ("CTDI"). This interface extends TDI to allow tabs to be dragged and dropped onto one another to form a "master tab" whose data page renders a "side by side" view of its combined "sub tabs". The combined view will revert to the previous form when closing the master tab. It's a novel solution for those websites that needs to compare information, such as e-commerce sites, online dictionaries, etc. To test the effectiveness of CTDI, we developed an online dictionary called "Engkoo" which puts the concept to practice.
\end{abstract}

Keywords: Information comparison, TDI, CTDI, AIC, tabs, combinable tabs.

\section{Introduction}

With so much information available through the internet, one of the major issues in computing today is designing a browsing user interface that not only models page style data but also makes the experience of navigating and interacting with the data feel natural. We went about looking at this issue by focusing on one major scenario common on the internet today: information comparison[1]. From our user studies, we found the majority of people go about finding their target information by sorting through numerous data by a process of identification and comparison. However, users are required to open a new page and must switch back and forth between the original pages if they want to compare, which is cumbersome to many users because of the repetitive action and sizable number of clicks. Our design practice is based on a project whose aim was to build a breakthrough online English-Chinese dictionary website for Chinese people called Engkoo. 


\section{Process}

We ran a user interview that contains 8 participants who frequently use online dictionary. Besides open questions, participants were also asked to show their "using" mode of online dictionary as they usually do and the procedure was tracked and recorded. A key finding from the interview, which is the underpinning of our Combinable Tabs work, can be coined "Appropriate Information Comparison" (AIC), as Figure1 shows. The AIC pattern was found when users needed to look up an English translation for a Chinese word. We observed the participants using the leading English-Chinese online dictionaries in Chinese market, Dict.cn, Youdao.com, and Iciba.com. For nearly all searched terms in these dictionaries, the results are multiple translations. We observed AIC when the participants copied and pasted the resulting translations, one by one, into the search box to execute further reverse dictionary look ups to verify their comprehension of the definitions and then switch back quickly between the tabs of the browser to compare the results of other definitions to find the most suitable one for their task, as Figure 1 shows. This user behavior pattern triggered us to think about how to support it in a more efficient way.

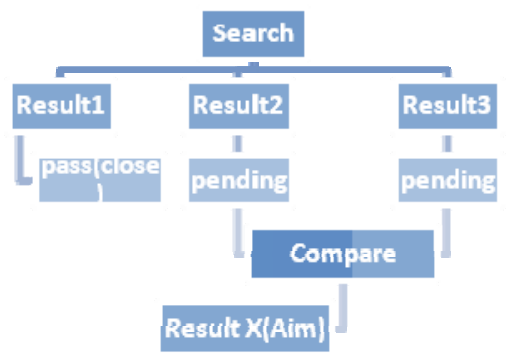

Fig. 1. AIC Model

\subsection{Engkoo Prototype 1}

We found that the users' requirement for comparison nearly always occurs when they search for synonyms or related words. The key intuition to prototype 1 is that these queries are always in sequential order. Therefore, our approach to enabling the entry point for comparison was to present the option for "compare" upon a subsequent search. The interface design was to enable the visibility of a compare button adjacent to the search button if another query is executed in the same session, as Figure 2 shows. When the compare button was clicked, the compare mode becomes activated, which presents a split view containing the definition results for the active query and the preceding query. Users can still type in new queries. When a new query typed and the compare button executed, it will be compared with the previous one, and the first one will be popped off. It is like a word comparison flow. The limitations with this approach are that it can't be used to compare non adjacent queries and also entering compare mode in this way isn't very intuitive. 


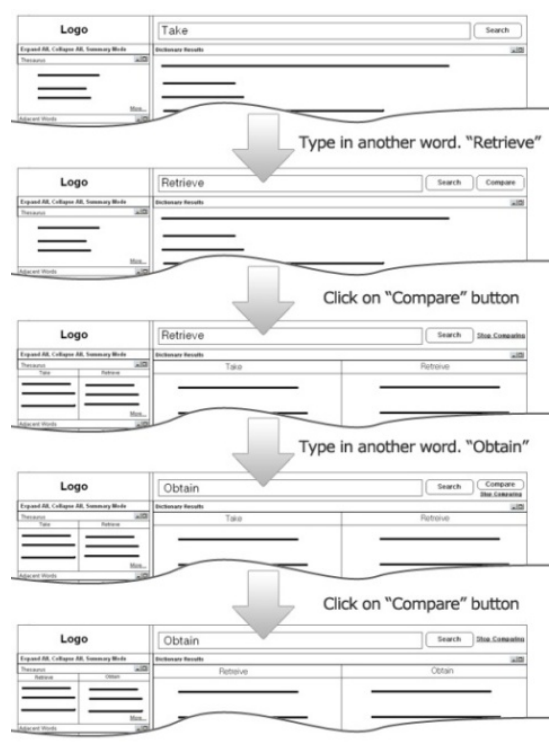

Fig. 2. Prototype 1

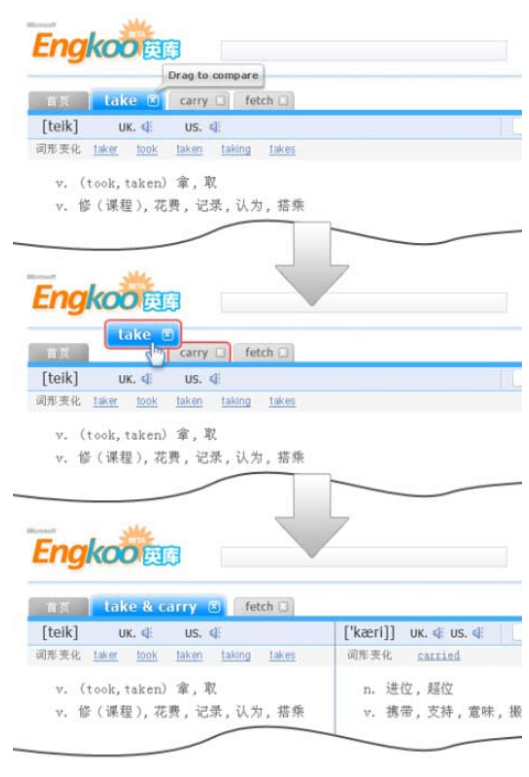

Fig. 3. Prototype 2

\subsection{Engkoo Prototype 2}

We brought the concept of a Tabbed Document Interface into our online dictionary design[2]. In our improved prototype 2, as Figure 3 shows, we innovated on the concept in an intuitive way by creating a Combinable Tabbed Document Interface which allows tabs to be dragged and dropped onto one another to form a master tab whose data page renders a side by side view of its combined sub tabs. When users click on an English translation to see the details (the same action as typing in a new query), a new tab is created. All the information of each tab, including definition, sample sentence, synonym, etc, is stored in the cache. Therefore, to the user, switching between tabs is an instantaneous operation. By just making the webpage TDI enabled - it would still carry all the same comparison usability problems generally associated with such an interface model. Our novel extension, CTDI, overcomes those usability problems by providing an intuitive method to enter into a comparison mode, which clearly shows the state is now in a compare mode, and allow for easy visual consumption of the information. To exit the comparison mode, users intuitively close the master tab and the sub tabs will be released into their previous state. One key benefit of this approach that it allows comparison of any two queries executed, not just the current and preceding ones as in prototype 1 .

\section{Evaluation and Findings}

For the evaluation, we design a task compared between our design and Iciba.com (\#1 market leader) as competitor. The task followed AIC process-(1)Search Chinese word “拿” in Engkoo.com and iCiba.com. (2)Explore the details of three translations and 
compare three of them at one time, such as "Take vs. Catch vs. Carry". (3)We recorded the "number of new pages" and "clicks" as a measurement of the usability [3]. The result shows that Engkoo.com has a obvious advantage in this task-"number of new pages":Engkoo(0), iCiba(3); “clicks":Engkoo(8),iCiba(17). Engkoo performs more effective and usable in the AIC process compared to iCiba-the market leader.

To design this new kind of interface for solving the AIC problem, this concept could be extended to a further pattern: internet search. Such a domain is analogous to the usability problems found in dictionary lookup because there is usually more than one result from a search query. Users click on the resulting hyperlinks that may or may not match the user's intended search[4]. Modern implementations of web browsers have partially made this task less arduous by implementing TDI; however the issue of not being able to simultaneously view the contents of multiple resulting web page documents is a usability inhibitor. A CTDI approach provides a solution to this problem while maintaining the benefits of a TDI interface.

\section{Conclusion and Future Work}

We have designed a novel concept to mitigate the AIC pattern by comparing information using CTDI. There is no need to have a "compare mode view" button or open another page for compare. All the action is executed in an intuitive way; drag to combine and close to end. To demonstrate this design we built a website prototype called Engkoo, which is publically accessible at http://www.engkoo.com.

In future work, we will explore the possibility of extending CTDI to all users to open multiple tabs at one time. A powerful aspect of this design is that users can combine $\mathrm{N}$ tabs together for comparison. That is, they can continually drop tabs into the master tab to compare further, which continually subdivides the visual area, and if necessary a horizontal bar is enabled to scroll if data is pushed off-screen.

\section{References}

1. Baeza, R., Ribeiro, B.: Modern Information Retrieval. Practical Comparison, 214-215

2. Maximilian, V., Carsten, S., Vassil, G., Michal, S.: Relevance and Impact of Tabbed Browsing Behavior on Web Usage Mining. In: WI 2006. IEEE Computer Society Press, Los Alamitos (2006)

3. Hornbæk, K.: Current practice in measuring usability: Challenges to usability studies and research. International Journal of Human-Computer Studies 64, 79-102 (2006)

4. Jan, C.W.: Single-source indexing. In: SIGDOC 2001. ACM Press, New York (2001) 